\title{
Upstream Experiments on the Gigabit PON Physical Medium Layer
}

\author{
D. Verhulst, Y. Yi, X.Z. Qiu, S. Verschuere, Z. Lou, P. Ossieur, J. Bauwelinck, X. Yin and J. Vandewege \\ Ghent University, INTEC/IMEC, Sint-Pietersnieuwstraat 41, 9000 Gent, Belgium. \\ dieter.verhulst@intec.ugent.be \\ B. De Vos \\ Alcatel Research \& Innovation, F.Wellesplein 1, 2018 Antwerpen, Belgium.
}

\begin{abstract}
First time demonstration of a high performance 1.25 Gbit/s GPON burst-mode uplink exceeding the ITU-T G.984 Recommendation and supporting Power Leveling Mechanism (PLM). A burst-mode receiver sensitivity of $-31.6 \mathrm{dBm}$ was achieved with a dynamic range of $21.9 \mathrm{~dB}$. (C) 2005 Optical Society of America

OCSIS codes: (060.4250) Networks; (060.4510) Optical communications
\end{abstract}

\section{Introduction}

Gigabit Passive Optical Networks (GPONs) following different FTTx scenarios (fiber-to-the- premises / cabinet / building / home / user) can offer efficient gigabit transport for "triple play" suites of voice, video, and data services. Guaranteed Quality of Service (QoS) is possible at a high bandwidth and transport efficiency. Fig. 1 shows the network architecture of a symmetric 1.25 Gbit/s GPON network optimized for variable-length packet transmission, and approaching the final demonstration phase within the European IST project GIANT (GIgapon Access NeTwork). In the GPON system, one fiber is shared by up to 32 Optical Network Units/Terminations (ONUs/ONTs) over maximum $20 \mathrm{~km}$ of cabling. The Optical Line Termination (OLT) broadcasts data in the downstream direction to all ONUs. The upstream data from an ONU is sent to the OLT in a Point-to-Multipoint (P2MP) Time Division Multiple Access (TDMA) scheme.

Due to the bursty nature of the traffic, the development of reliable upstream Physical Media Dependent (PMD) prototypes forming a GPON upstream link is a challenge. To our knowledge there are no real burst-mode PMD components available on the open market, that are suitable for a high performance GPON uplink. The complete upstream link described in this paper was developed and tested successfully within the GIANT project, and is based on newly-designed burst-mode PMD chips provided by the Belgian IWT project "SYMPATHI" (SYMmetrical PON AT HIgh bit rate) [1]. The experimental results are fully compliant to the ITU-T Recommendations G.984.2 specifying the GPON physical medium layer and G.984.3 specifying the transmission convergence layer.

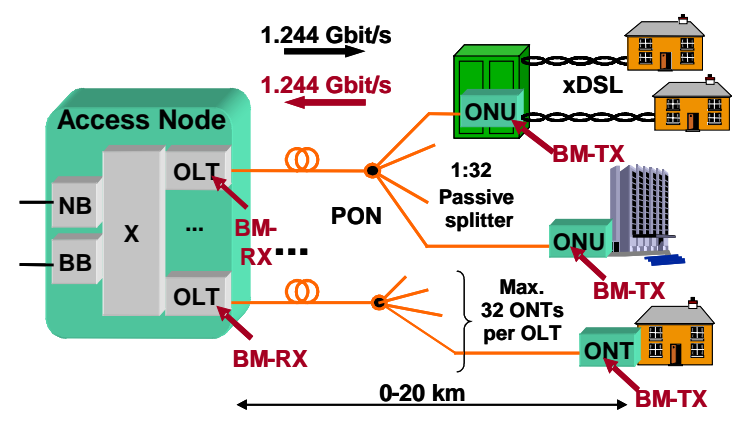

Fig. 1. The gigabit PON network architecture for different FTTx scenarios

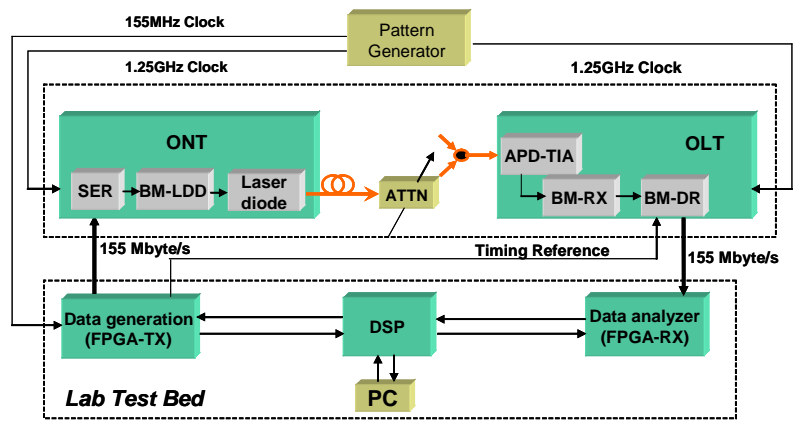

Fig. 2. The GPON physical media layer uplink prototypes and test set-up

\section{Burst mode PMD prototypes}

Fig. 2 depicts the GPON physical media layer uplink under test. It mainly consists of Upstream Transmitters (USTX) at each ONU site, and an Upstream Receiver (US-RX) followed by Burst-Mode Data Recovery (BM-DR) at the OLT side. Each US-TX contains a laser diode and a Burst-Mode Laser Diode Driver (BM-LDD). The 155 Mbyte/s parallel data inputs from an FPGA burst pattern generator are serialized to generate both the 1.25 Gbit/s data stream and the burst envelope signal driving the US-TX. To avoid collisions of bursts originating from different ONUs, the 
ONU Serializer (SER) adds a programmable delay with bit accuracy until the launched bursts are aligned with the ongoing traffic at the OLT. An ONU can only transmit in the time slots assigned to it by the OLT. An instant laser shut-down within the BM-LDD ensures that the ONU does not transmit any power in slots assigned to other ONUs. The BM-LDD also contains a fast but accurate digital Automatic Power Control (APC) algorithm to regulate and stabilize the launched optical power of the US-TX [2]. The optical power can be set to three levels within a $6 \mathrm{~dB}$ range to support the optional Power Leveling Mechanism (PLM) defined in ITU-T G984.2. This reduces the dynamic range requirement of the OLT receiver, extends the laser lifetime, reduces optical reflections and decreases the power consumption of the ONU.

The 1.25 Gbit/s burst-mode US-RX contains a high sensitivity APD Transimpedance Amplifier (APD-TIA) and a wide dynamic range Burst-Mode Receiver (BM-RX) designed for instantaneous burst amplitude recovery [3, 4]. It receives optical packets from all active ONUs in very fast succession, with varying signal level and phase from packet to packet. The packets are interleaved with a guard time of 4 bytes at $1244 \mathrm{Mbit} / \mathrm{s}$ (or $25.6 \mathrm{ns)}$ ) as specified in G984.2 and shown in Fig.3. The combination of $21 \mathrm{~dB}$ level variation, a short guard time and a maximum of 72 consecutive identical bits within a packet requires the US-RX to be dc-coupled with automatic offset compensation. A BM-RX requires fast threshold extraction on individual incoming packets to perform dynamic level detection and amplitude recovery. The BM-RX we designed accurately extracts the decision threshold within a preamble length of 3 bytes (12 bits " 1 " and 12 bits "0") at the beginning of each packet. It also contains circuits for ONU initialization and for optical power measurements supporting the PLM.

As the ONU upstream data clock is extracted form the incoming downstream data, no upstream clock recovery is needed at the OLT: a sample of the $1.25 \mathrm{GHz}$ OLT downstream clock is used for the upstream data recovery. The bit recovery function in the BM-DR is based on over sampling with digital phase selection. No memory is used to store phase information of previous bursts from the same ONU. At the beginning of each burst, the phase selection algorithm exploits a 24-bit CPA (Clock Phase Alignment) field filled with "101010" pattern in the overhead containing maximum transitions to lock to the phase of the incoming data (see Fig.3). Once the bit recovery is in lock it starts tracking the data to compensate for possible small phase drifts during the payload of the packet. The delimiter detection circuit in the BM-DR is used for both byte recovery and delay measurement. One of the functions of the OLT is to divide the upstream data link into time slots and assign then to the ONUs. The OLT knows when every burst will arrive. The BM-DR gets this information via a Timing Reference (TR) input signal. The delay between the pulse on the TR input and the position of the first bit of the delimiter is calculated and reported over a dedicated interface.

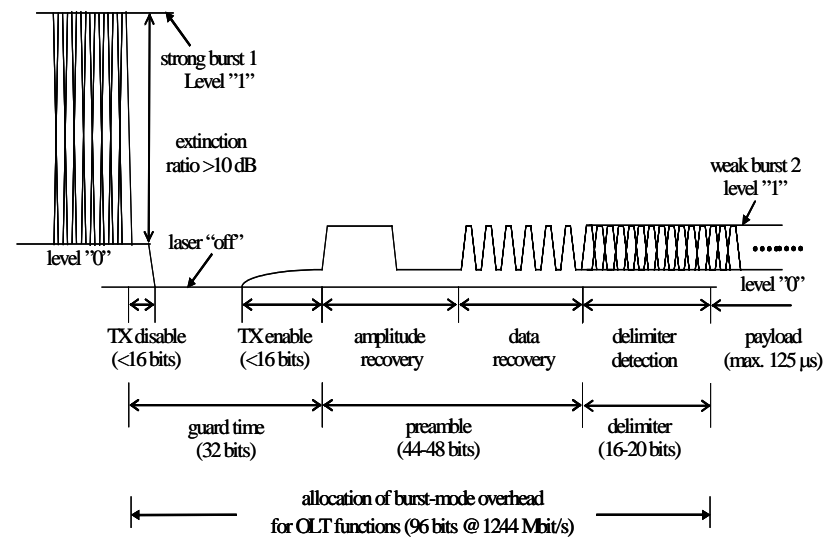

Fig. 3. Illustration of input signals at OLT with an upstream overhead (96 bits in total)

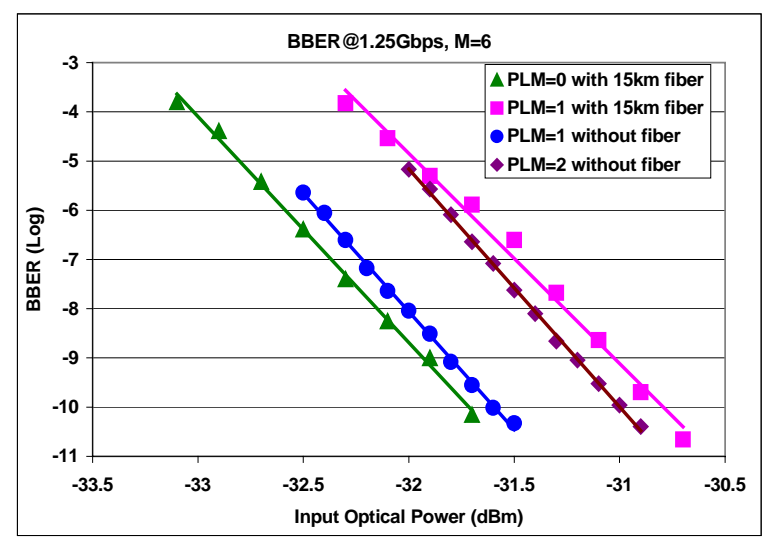

Fig. 4. BER test results on the complete uplink including BM-DR $(\mathrm{M}=6)$

\section{Experimental results}

A GPON lab test bed was built to evaluate the burst-mode packet transmission over an upstream optical link in the INTEC_design laboratory of Ghent University. The experiments have been performed in three steps. For the first two steps a high-speed burst-mode BER (Bit Error Ratio) tester was deployed to generate the upstream data and measure bit errors with a built-in data analyzer module. First of all a back-to-back uplink containing only the US-TX and US-RX was established and tested to find the BER baseline. A sensitivity of $-32.8 \mathrm{dBm}$ with a dynamic range of $23 \mathrm{~dB}$ was achieved at $\mathrm{BER}=10^{-10}$ and $\mathrm{APD}$ gain setting $=6$ [5]. 
Secondly two US-TX modules were used to generate a strong packet $(-10 \mathrm{dBm})$ followed by a weak packet, emulating a worst-case scenario at the US-RX as shown in Fig.3. An US-RX sensitivity of $-30.6 \mathrm{dBm}$ was measured combined with a dynamic range of $21.6 \mathrm{~dB}$. So $2 \mathrm{~dB}$ worst-case burst-mode receiver sensitivity penalty was found. This is better than the G.984.2 specification of - $28 \mathrm{dBm}$ (with a 1.3/1.5 $\mu \mathrm{m}$ WDM) as listed in Table 1. Major cause of this burst-mode sensitivity penalty is the turn-off tail from APD and TIA chip conflicting with the strict requirements of short guard time and preamble length. This penalty could be reduced if we would have a guard time of 6-8 bytes.

Table 1. Performance parameters for the upstream link

\begin{tabular}{|l|c|c|c|}
\hline Parameter & $\begin{array}{c}\text { Measured } \\
\text { (without PLM) }\end{array}$ & Spec. & Unit \\
\hline bit rate & 1.244 & 1.244 & Gbit/s \\
\hline ODN (Class B) & $\begin{array}{c}>15 \mathrm{~dB} \\
\text { differential range }\end{array}$ & $\begin{array}{c}\text { min.10 } \\
\text { max. } 25\end{array}$ & $\mathrm{~dB}$ \\
\hline $\begin{array}{l}\text { mean launched power } \\
\text { (without PLM) }\end{array}$ & $\begin{array}{c}1 \mathrm{~dB} \text { tolerance } \\
\text { over -40-85 C }\end{array}$ & $\begin{array}{c}\text { min. }-2 \\
\text { max. }+3\end{array}$ & $\mathrm{dBm}$ \\
\hline extinction ratio & $>10$ & 10 & $\mathrm{~dB}$ \\
\hline $\begin{array}{l}\text { consecutive identical } \\
\text { digit immunity }\end{array}$ & 72 & 72 & $\mathrm{bit}$ \\
\hline receiver sensitivity & $\begin{array}{c}-31.6 \\
\text { (w/o WDM) }\end{array}$ & $\begin{array}{c}-28 \\
\text { (with WDM) }\end{array}$ & $\mathrm{dBm}$ \\
\hline receiver overload & $\begin{array}{c}-9.7 \\
\text { (w/o WDM) }\end{array}$ & -7 & $\mathrm{dBm}$ \\
\hline dynamic range & 21.9 & 21 & $\mathrm{~dB}$ \\
\hline Overhead & 12 & 12 & byte \\
\hline guard time & 4 & 4 & byte \\
\hline
\end{tabular}

Table 2. Burst-mode BER test results with the PLM

\begin{tabular}{|l|c|c|c|}
\hline Parameter & PLM=0 & PLM=1 & PLM=2 \\
\hline $\begin{array}{l}\text { peak launched power / } \\
\text { bias level (dBm) }\end{array}$ & $3.5 /-13.5$ & $0.5 /-13.5$ & $-2.5 /-13.5$ \\
\hline extinction ratio (dB) & 17 & 14 & 11 \\
\hline $\begin{array}{l}\text { receiver sensitivity } \\
\text { (no fiber) }\end{array}$ & $\begin{array}{l}\text { not the case } \\
\text { (PLM=1, 2) }\end{array}$ & $-31.6 \mathrm{dBm}$ & $-30.9 \mathrm{dBm}$ \\
\hline $\begin{array}{l}\text { receiver sensitivity } \\
(15 \mathrm{~km} \text { fiber) }\end{array}$ & $-31.7 \mathrm{dBm}$ & $-30.7 \mathrm{dBm}$ & $\begin{array}{l}\text { not the case } \\
\text { (PLM=0, 1) }\end{array}$ \\
\hline
\end{tabular}

Remark: Data generation respects the GPON standard, with 4 bytes guard time between two bursts followed by a preamble of 12 bits of " 1 " and 12 bits of " 0 " for the RX amplitude recovery, a 24 bits CPA field filled with a ' 1010 ' pattern and a 16 bit delimiter. The payload of the packet is a $2^{15}-1$ PRBS sequence. The APD gain setting $M=6$. The PLM=1, 2 operates only when an ONU experiences a low ODN loss to avoid overload of the US-RX. The PLM mode can be set locally via an SPI interface under the BM-RX control via the GPON downstream link.

In the last test phase, an uplink including also the BM-DR at the OLT and the serializer at the ONU was tested at different PLM modes. The PLM allows each ONU to operate in three discrete output power modes, with following mean launched power: 1 ) normal mode minimum and maximum $=-2 /+3 \mathrm{dBm}$ as stated in Table 1 ; 2) mode $1=$ normal - 3dB; 3) mode $2=$ normal - 6dB. For these experiments an FPGA-based test platform ("Lab Test Bed", see Fig.2) was developed to generate the burst data (FPGA-TX) and test the burst BER (FPGA-RX). Fig. 4 shows a set of measured BER curves, and the test results are summarized in Table 2. Therefore, a conclusion can be made that 1) with $15 \mathrm{~km}$ fiber, by increasing the extinction ratio from 14 to $17 \mathrm{~dB}$, the receiver sensitivity can be improved by $1 \mathrm{~dB}$; 2) without $15 \mathrm{~km}$ fiber, the receiver sensitivity improvement was $0.7 \mathrm{~dB}$ when the extinction ratio increased from 11 to $14 \mathrm{~dB}$. Furthermore, when the PLM mode was set to 1 , the receiver sensitivity was degraded by $0.9 \mathrm{~dB}$ due to the insertion of $15 \mathrm{~km}$ fiber.

\section{Conclusion}

A high performance 1.25 Gbit/s upstream link for a Gigabit PON physical layer has been successfully integrated with a complete set of innovative burst-mode PMD prototypes, which is publicly demonstrated for the first time. The test results exceed the tough specifications of the newly approved GPON ITU-T recommendation G.984.2. A burst-mode receiver sensitivity of $-31.6 \mathrm{dBm}\left(\mathrm{BER}=10^{-10}\right)$ with a wide dynamic range of $21.9 \mathrm{~dB}$ were simultaneously achieved at an APD gain setting of 6, offering excellent flexibility for network deployment.

\section{References}

[1] X.Z. Qiu, P. Ossieur, J. Bauwelinck, Y. Yi, D. Verhulst, J. Vandewege, B. De Vos, P. Solina, “Development of GPON Upstream Physical Media Dependent Layer Prototypes”, to be published by IEEE JLT (Nov. 2004).

[2] J. Bauwelinck, D. Verhulst, P. Ossieur, X.Z. Qiu, J. Vandewege and B. De Vos, "DC-coupled burst-mode transmitter for a 1.25 Gbit/s upstream PON”, IEE EL 40, 501-502 (2004).

[3] P. Ossieur, Y. Yi, J. Bauwelinck, X.Z. Qiu, J. Vandewege and E. Gilon, “DC-coupled 1.25 Gbit/s burst-mode receiver with automatic offset compensation”, IEE EL 40, 447-448 (2004).

[4] P. Ossieur, X. Z. Qiu, J. Bauwelinck, J. Vandewege, "Sensitivity Penalty Calculation for Burst-Mode Receivers using Avalanche Photodiodes", IEEE JLT 21, 2565-2575 (2003).

[5] X.Z. Qiu, P. Ossieur, J. Bauwelinck, Y.C. Yi, D. Verhulst, S. Verschuere, Z. Lou, W. Chen, Y. Martens, X. Yin, J. Vandewege, B. De Vos, E. Gilon, "FSAN GPON upstream burst-mode Transmission Experiments", in Proceedings of $30^{\text {th }}$ European Conference on Optical Communication, Stockholm, 2004, 398-399. 